ISSN 1392-1541

Geodezija ir kartografija, 2005, XXXI t., Nr. 2 Geodesy and Cartography, 2005, Vol XXXI, No 2

UDK 528.9

\title{
LITHUANIAN GEOGRAPHIC INFORMATION INFRASTRUCTURE - SPATIAL DATA SHARING WITH LITHUANIA
}

\author{
Cindy Pubellier \\ Geolines, 5 Place des Brugnauts, 92220 Bagneux, France +33 (0)663719162+66 (0) 145366872 \\ E-mail: cpubellier@geolines.tk
}

Received 1711 2004, accepted 06042005

\begin{abstract}
Geographic information system (GIS) has proved its success in the past decades by its wide utilisation bringing information together within organisations. Nevertheless, our challenge now is to use and share spatial data to its maximum advantage within the IT (Information Technology) environment instead of merely using GIS. This integration or interoperability of data is essential to information and knowledge sharing within a nation. Technology is undoubtedly crucial for the vision of National Geographic Information Infrastructure (NGII) to make interoperability happen through which tremendous economic benefits could be achieved. Of equal importance is a clear framework of agreements among government agencies, and between the government, the private sector and citizens on the terms through which the use of PSI, including geographic information, can be maximised for the benefits of all. These agreements often require attention and political support at the highest level.
\end{abstract}

Keywords: Geographic information system, Geographic Information Infrastructure, Spatial Data Infrastructure.

\section{Introduction}

Geographic Information (GI) has tremendous economic and social value for any society. GI is very expensive to collect process and maintain, yet ever easier to disseminate cheaply via Web-based services and products. Studies from developed nations around the world show that GI plays a crucial role in underpinning whole economies and delivering efficient government, indicating that it should be used as widely as possible. Much GI is collected by local and national government for specific purposes. How such Public Sector Information (PSI) is made more widely available for other uses and to other users and at what price has created heated debate and led to the adoption of diverse PSI changing policies in different countries.

The wide utilisation of GIS software, the high production of GI and the exchanges of data among institutions but yet insufficient geospatial data re-use in Lithuania have witnessed the increasing demand for a more organised mechanism and infrastructure to benefit the whole country and ultimately to Europe and the rest of the world.

One of the goals of LGII (Lithuania Geographic Information Infrastructure) is an open, shared infrastructure for accessing and distributing information products and services online $[1,2]$. To achieve this goal, data residing at regional and national organisations are to be implemented to common standards.

\section{Objectives of LGII}

One of the most valuable resources of a nation is its data. Not only the need for data sharing is important for everyday business processes, but it shows its value in decision-making phases during natural disasters or manmade emergencies where efficiency means life and property savings. The main aim of LGII is to develop an official Geographic Information source system or Infrastructure and Information Environment allowing the free incorporation of GI into almost any Public Sector Information (PSI) Services in Lithuania [1].

The overall goal of LGII is to improve the quality of policy making, citizen's participation and market development through the increased use of GI, hence to provide a new means of acquiring knowledge.

It is central to developing a sustainable national SDI to foster and to maintain relations of trust among all stakeholders (citizens, public sector, private sector and research). To achieve this goal, we need to encourage the collection, processing, archiving, integrating, and sharing of geospatial data and information using common standards and interoperable systems and techniques through the Internet [2-10].

The objectives of the project are to [1]:

- $\quad$ Provide GI (as part of PSI), prime digital material for almost any public sector information services, research, business and citizens at large - $\underline{\text { II }}$ availability via the Internet 
- Combine/interconnect the GI prime material data into different sources of added value electronic public sector products and services, hence leads to unprecedented possibilities to interconnect various register, databases, etc. - Interoperability

- Foster substantial investment in creativity and innovation, and lead, in turn, to growth and increased competitiveness in both content provision and Information Technology and more generally across a wide range of industrial and cultural sectors. This will safeguard employment and encourage new job creation - Economic gain

- Provide transparent and applicable environment for the re-use of GI, without which is a major barrier to bring out the full economic potential of this key information resource (There are considerable differences in the rules and practices relating to the exploitation of the current insufficient and fragmented available GI resources) - favourable conditions for business and industry

The purposes of LGII can be summarised as follows:

- Identify both users and providers of spatial data in order to create an infrastructure that meets everybody's needs;

- Encourage the development of partnerships, for creating geographic data for the various programmes in government, academic institutions and the private sector;

- Increase usability of existing GI. Provide an environment for users to share their information, knowledge and interests by easy access to good quality spatial data. In this way, through consensus, an appropriate infrastructure can be established;

- Encourage connections and coordination among government institutions, the private and educational sectors through sharing of spatial data. The cooperation of organisations shall be intensified by the pulling together representatives from sectors of data production, research and education as well as the business sectors using spatial data.

Geographic information is a perspective to the Information Society and the general information infrastructure than just a separate sector of information technology.

\section{The Feasibility Study}

LGII is not merely about technology, but about developing a clear framework of agreements among government agencies, and between the government, the private sector and citizens on the terms through which the use of PSI, including geographic information, can be maximised for the benefits of all. These agreements often require attention and political support at the highest level.
A thorough Feasibility Study financed by the European Union, commenced on March 2004, has been conducted by a team of international and local experts in the GIS-Centras, Vilnius, under the Ministry of Agriculture. Interviews, questionnaires, presentations are conducted during the course of this phase, to promote the vision of LGII and to gain support and involvements from stakeholders. Though at a pre-project stage, most of the GI users and providers are favourable towards LGII, while some others are still unaware of the benefits and reluctant to change or due to their satisfying financial situation as islands. Consequently, promotion to raise awareness has to be continued. It has to be pointed out that LGII is to them a new concept which is not yet tangible. Promotions and presentation as soon as LGII becomes the reality will be much more effective and efficient.

\section{Project results}

The project will result in substantial investment in creativity and innovation by the provision of digital content in geographic forms through which knowledge can be acquired. Moreover, the project opens possibilities for new services and products creation. Figure illustrates various areas of activities and expected outcomes of LGII Development Project.

Metadata and Data Publishing. "GIS search engines" will be available to search the catalogues of producers and custodians. These catalogues should provide a standardised view on metadata. The issue of metadata is vital to the success of LGII. Metadata are to be accessible through the LGII portal (or clearinghouse) as soon as they are ready in the project implementation. This way, existing data can be accessible sooner in a socio-economical advantageous manner.

Geographic Information Finding/Viewing. By means of a data locator, users are able to discover geospatial information or related services.

Delivery of GI. When the user needs to be in possession of the data presented in the internet, delivery of data over the Internet should be available. Different modes can be envisaged (via e-mail, through web feature server, off-line media such as tape and DVD for data of large volumes, etc).

Possibility of Analysing GI interactively. A number of applications can be realised by combining a series of geospatial services and spatial queries in appropriate workflows over the Internet, without having to store the thematic reference information locally. 


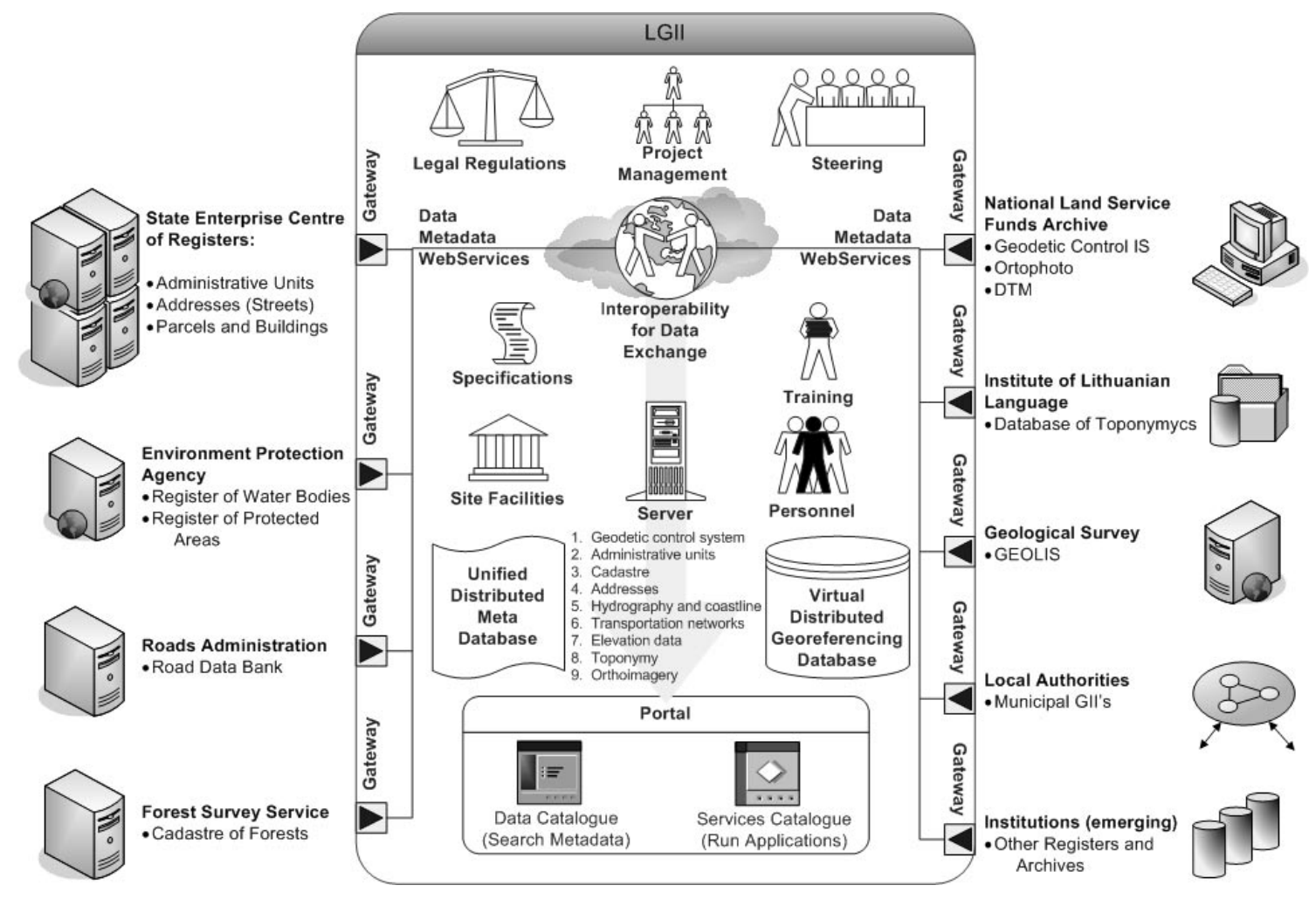

Area of activities and expected outcomes of LGII Development Project

\section{Risks and flexibility}

\section{Change of organisational cultures}

The management of change in organisation, working relationships among networks of organisations are new challenges to be faced by LGII. Merely passing laws is not sufficient if the detailed work is not undertaken and developing programmes are not moving on with a stepby-step strategy. The required capacities do not need to be centralised in the form of a large organisation. Rather the contrary, experience shows that a small group with clear political support may work in a decentralised way. The success of this management of change depends on the commitment of those responsible for implementing the change itself.

The development of LGII needs to include education and the change of organisational cultures. These are often lengthy processes for many public sector organisations that have difficulty in adapting quickly to change. Some of challenges that need facing include the need to work more horizontally across departments and agencies, having a greater sensitivity to customer needs and requirements, and using information more effectively as well as the ability to let go to my information. We are therefore talking about long-term processes that may take 20 or more years. However, LGII does not need to wait until such changes take place. Rather the contrary, they can be used as an opportunity to leverage change.

Constant feedback and evaluation with indicators are essential from political and managerial points of view.
They act as assessment tools for both political and managerial visibilities.

\section{Underestimation of capacity building}

Insufficient reflection given to the need for capacity building (education, training) and targeted research throughout the deployment could be a problem facing the sustainability of LGII. There is often a lack of awareness at local level of the importance of data sharing. It is of prime importance to focus on the benefits derived from the use of GI.

The viable long-term strategy is to maintain a balance between the development of management capacities and the acquisition of LGII policy responsibilities. Initial progress is liable to be slow; however, a succession of challenge-response cycle leads to an enhanced performance without causing excessive stress or destructive overload. Making haste slowly has less obvious political appeal than the promise of quick results but it seems likely to provide a firmer foundation for long-term effectiveness.

\section{Difficulties to pulling people to work together}

The different nature of work and traditional responsibilities of stakeholders and beneficiaries makes this project complex. They did not have experience in working together. Thus a coordinator is an absolute necessity to foster the cooperation among different institutes. 
Success depends also on qualified management, sustained concerted effort and well-organised teamwork. A Core Technical Advisory Board/Committee with European experts should be established, in close contact with their European (ESDI) counterpart, to define specifications and provide technical coordination and advice. A mandatory body responsible for coordination is central in this project.

\section{"Departmentalism"}

Any organisational change in government commences with high expectations and may results in dismay and disillusionment especially with bureaucratic resistance to change. To get around the problem, every effort should be made to identify the main sources of resistance and to anticipate and formulate ways to deal with them. Mere political will is necessary but not sufficient to guarantee the success of reforms.

Processes, feedback and evaluation are imperative both politically and administratively. Progress can be assessed by the coordinators and managers with clear visibilities. Feedback and evaluation provide indications for maintaining internal and external support.

\section{GI community as a minority}

Like in many countries, GI specialists are still a very small minority of the whole population in Lithuania although the Geo-spatial community is growing every year. Special training and education courses should be done to reduce the natural resistance to unknown subjects especially GI is a complex issue with its potential to integrate institutions and administrative units. Cooperation and partnership are of vital importance to overcome traditional hurdles and to exploit the full potential of LGII.

Promotion and capacity building are the only means that can raise the awareness of all. Communication between the GI community and the non-GI community through workshops, conferences, seminars etc should be conducted. By doing so, people will think about using GI as a reflex when needed. The private sector should play an important role not only in data usages but also in data collection through the production of value-added products and services.

\section{High investment cost}

The high investment cost is definitely a risk for any NSDI. Indeed, economic benefit will only show later in the project. Nevertheless, an economic analysis conducted during the Feasibility Study shows that the ECONOMIC internal rate of return of the investment (ERR) estimated for a time horizon of 10 years, to be of a high value demonstrating that the external benefits to the society as a whole are much compensated even with the high investment cost in the beginning.

\section{Benefits of LGII}

Like all SDIs across the world, the costs of implementation are front-loaded while the benefits only start showing at a later stage. That is why every effort should be made to demonstrate some early results through an iterative process resulting in evolutionary acquisition and deployment. It is widely recognised that the quantification of the benefits of Spatial Data Infrastructure to be particularly challenging.

Despite the fact that some benefits are easily identifiable and can be quantified, others are much intangible, especially the provision of information is a means to different ends which are results of many parameters. In such cases, it is impossible to identify completely and evaluate the benefits.

LGII should be developed at local, regional, national, European and global levels. Consequently, there is a need to address politicians and decision-makers at each of these levels and demonstrate its benefits.

The availability of public information is an absolute prerequisite for the competitiveness of a society. The difficulties in searching for fragmented information are considered to have a negative bearing on job creation. A study conducted for the European Commission estimated the economic value of PSI in Europe at 60-70 billion Euros per year, or which over a half was accounted for by GI (mapping, land and property, utilities, environmental data, ...) GI is definitely a fundamental element of PSI.

The benefits include domains of high political priority such as crime reduction, health, education, spatial planning, environmental protection and risk management. Moreover, LGII helps supporting egovernment and general economic development, reduce duplication and waste resources and increase competitiveness through the development of new industries such as the location-based services. LGII.

There can be direct and indirect effects to implement

Some Direct effects entail efficiency gain for governments, business and citizens; cost reduction in data purchasing (via the web instead of through agreements and direct purchase); less expenditure in datamanagement due to specialisation; less expenditure in reuse of data; having a common and standardised set of indicators about the state of the environment, thus being able to document the success of a policy towards sustainable development and further on becoming able to compare sustainable development at the national level.

Other Indirect effects include reducing the burden of taxation for citizens, companies and governments; making better decisions at governments and companies; developing new applications (product-innovation and market-development)

\section{Benefits for the users}

Standards solve problems such as the way to represent data efficiently and create benefits like interoperability, portability, ease of use, expanded choice and economies of scales. There will be efficiency gain 
for the users of LGII due to standardisation (better portability of data): interoperable specifications reduce the costs of combining datasets. In absence of standardisation, many projects will require the expenditure of time and money in order to secure the oneoff conversion of one or more datasets. Otherwise, work will not be done due to the data conversion cost which is excessive. Standardised metadata: reduces data collection cost; expansion of production of new activities that would have been too expensive if total creation is needed. Efficiency gain is also realised through easier access ,hence ability to discover existing datasets reduces the access barriers. Users work much faster due to known value of the data and better quality of the data. Consequently, better decision-making and more economical planning can be envisaged due to lower pricing of the data (multi-use). Economic advantages can be derived from coordination: sustained commitment, vision and support to all the stakeholders involved should be maintained to keep the project focused and built on the achievements.

\section{Benefits for the data providers}

Efficiency gain can be accomplished due to just once collection of data (less costs of collecting); due to back-office reorganisation (simplification of procedures with coordinated e-government portal solutions; due to a more efficient use of personnel (specialisation); due to the use of standards (economies of defining data models); due to more use (hence more valued) of the offered data; due to easier exposure of available data (wider market)

\section{Benefits for the Government in general:}

- Compliance ith PSI directive and enabling PSI (including registries) interoperability;

- Driving force of e-government and knowledge-based economy;

- Efficiency gain on environmental impact assessment, environmental monitoring and protection;

- Better policy on risk prevention, health and environment.

\section{Benefits for the GI industry:}

- Developing products that fit the European standard on Geo-information and make them available for a wide pan-European market.

- Knowledge is available for searching, accessing and using geo-information.

Benefits (cohesion policies) for other interventions at the European level

- INSPIRE, GMES, eEurope and many other measures will benefit from the implementation of LGII so that environmental policies and planning at the national level and hence European Level will be better made as a result of better decision-making based on better GI.

Increase in real estate transaction. LGII encourages exploration of land properties and monitoring the use of land, creation and introduction of the system of territorial planning of the organisation of land exploitation. Given the real estate market in Lithuania to be $434 \mathrm{~m}$ EUR in 2002, an increase in 1\% through LGII is reasonable. Today home buyers and real estate investors have no real tools to explore potential parcels but only through parcel numbers or predefined zonings. LGII will provide additional entry points from environmental and transport scenes. International investors can find a golf site where it is topographically and environmentally (forest, river) suitable for the purpose and not too far away from a particular place. Home buyers can locate a place where public and road transport to be good according to their family composition.

\section{Socio-cultural benefits}

In terms of its support for spatial policy-making, its criticality to national security and emergency management and in terms of its inherent value.

\section{Tourism}

LGII can, with time, create an image of Lithuania as an attractive tourism country starting with a comprehensive and easy access of transport data.

\section{Good governance}

LGII also will benefit citizens in facilitating access to good and consistent GI that helps in making key decisions such as home purchasing, including factors of flooding, proximity to industrial sites, transport, health care, education facilities, etc that will raise the citizens' quality of life.

LGII will enable public sector information (PSI) to be exploited by private sector with a view to the creation of added value services that are useful to the public and resulting in the creation of high quality employment (as in the US).

LGII will also foster the public participation of decision-making on specific activities.

\section{Assessment of LGII from the financial and economic perspective}

Lithuania's scarce natural resources stimulate the mobilisation of other resources that are important and promising under current conditions such as human resources, innovative activity and knowledge-based production. LGII is undoubtedly a long-term solution to efficiency and effectiveness from the financial and economic point of view.

Lithuania has the prerequisites necessary for the rapid technological progress such as applied sciences' potential and resources for synthesising the knowledge from different fields of activity, it produces some hightech products and renders high-tech services, its industry uses many fundamental technologies and the country's industrial and scientific concentration is satisfactory. By 
providing a knowledge-based environment, not only does the public sector benefit from efficiency gain by economies of scale and specialisation, production of data will be facilitated using new management techniques and specifications through LGII. The information age means that the need to spend days seeking out data from other public sector organisation, to spend hours checking the quality of the data or adapting the data to the specifications used in their own report should be ceased to continue. State registers and databases should be operated within the general state data collection system such as LGII. The state would be incapable of performing its functions without improving its administrative skills. Good administration and transparent activities of the government are the main conditions for the country's rapid progress. In this respect, LGII will enable the increase in competitiveness of goods and services of all branches of economy starting from the public sector.

As a small country, Lithuania has a capacity of promptly adapting to the rapidly changing economic globalisation circumstances and fast spreading of means of informatics in the economy. Lithuania has a comparatively good fixed and mobile communication infrastructure, the broadband Internet and cable television services, qualified informatics specialists, an advanced specialist training system and the first positive elements of the distance education structure. The majority of the Lithuanian population thinks that information technologies will have a favourable impact on the economy of Lithuania. Businessmen envisage the significance in the enhancement of the production of information technologies and telecommunication products as well as provision of the related services. LGII stimulates business and fosters job creations through new products and services, especially for small and mediumsized business, as a result of easy access to GI by the whole community. Small-and medium-size business sector has a progressive infrastructure, operates in the open and gradually developing economy, uses modern technologies, skilled labour force, manufactures competitive and innovative goods and provides services to the local and international markets, thus forming a better part of GDP. Small-and medium-size business can flexibly adjust to the changes in the market, reasonably uses resources and creates new workplaces.

LGII as a part of Information Technology is a knowledge-gathering activity by its geographic nature. It is one of the effective components of the physical and economic infrastructure ensuring stable and balanced development of the economy and its efficient functioning.

Investments in LGII are crucial for economic development on both a national and an international scale due to the wide reaching inter-sectoral effects of improved efficiency in communications and the development of online services. It has a considerable influence in more general fields of civil development such as training, youth education, culture, tourism, politics etc.

\section{Conclusions and proposals}

The rapid increase in the number of countries becoming involved in National GII development are the fruits of lessons learned from the clear benefits of first generation of national GII (Australia, Canada, China, Denmark, Finland, France, Germany, Japan, Netherlands, Portugal, Switzerland, UK and USA). Their initial motivations were in reducing duplication, using resources more effectively, and creating a base from which to expand industry productivity and the spatial information market. The second generation countries started developing their national GII aiming at facilitating the planning and management of economic development and natural resources. Many evaluations derived from experience indicate that there was a lot of diversity in the first generation of national geographic information strategies. Up to now, there were clear advantages associated with a formal mandate for a national geographic information strategy, providing that this was accompanied by necessary resources to enable its implementation. Lack of dedicated resources is definitely a weak point, where initiatives are fundamentally results of existing coordination activities. This model has the advantage that it builds upon existing cooperative patterns.

In a way, one can say that Lithuania is very fortunate to be able to start LGII now so that the experience from the first generation National GII are learnt and mistakes can be avoided. Lithuania will be participating what we call the third generation national GII.

Even if we don't do it, others will. It is estimated that there will be a considerable increase in the number of national geographic information strategies being implemented through the world in the next ten years. The issue for all the countries is not to ask the question whether a National GII should be implemented, the challenge is how to implement it in an effective way basing on the culture, specific requirements, priority and history of the country to promote economic development, to stimulate better government and to foster environmental sustainability.

The "next thing," which we see already happening, is the emergence and importance of the Internet in GIS application. The "next thing", which people in the industry have been predicting for a while, is the use of GIS by people who don't need to understand the technology or the science. This broadens the user's base and deepens the importance of GIS. LGII gives more emphasis on accuracy, semantics, reliability, and control of geographic information. It opens the world to new opportunities, applications, and possibilities.

There is a need to integrate the socially excluded and special needs children to get access to reliable Geographic Information to acquire, develop and improve their knowledge and skills from either rural or urban areas especially in this ever-changing environment nowadays. Information that are accessible should not be limited to textual form but should extend to geographic so that a comprehensive vision is provided as " $a$ picture 
is worth a thousand words" applies to everybody. LGII strengthens the provision of Information Society by integrating all the public sector information that is tied geographically (geo-referenced).

LGII is also a means to minimise urban and rural information access differences so that all the population have equal opportunities to reach information for social and public needs.

A knowledge-based economy has a strong impact on the life of all citizens. It is a powerful engine for growth, competitiveness and jobs whilst improving citizens' quality of life. Economy based on knowledge is becoming one of Lithuania's priorities. The EU has set a goal to create a society based on knowledge within the nearest decade; in 2015, Lithuania may reach a similar level in this sphere. A society based on knowledge is a very important challenge to Lithuania and Lithuania may even fail in achieving it should no consensus is reached between wide sets of society and a strong intellectual political will.

\section{References}

1. Lithuanian Geographic Information Infrastructure. Framework Report. ASTEC Global Consultancy, June 2004 (draft).

2. EC-INSPIRE: Spatial Data Infrastructures in Europe: State of Play Spring 2003 Lithuania (draft).

3. Kennedy, S. Pipelines Benefits by Integrating Management Systems. Pipeline \& Gas Journal, Sept 2002.
4. „Online availability of public services: How Europe is progressing?". Cap Gemini Ernst \& Young's, Oct 2003 (draft).

5. UN-ECE 1996, Land administration Guidelines - Meeting of officials on land administration, UN Economic Commission for Europe (draft).

6. ANZLIC Benefits Study - Australian Land and Geographic Data Infrastructure. Price Waterhouse, Feb 1995 (draft).

7. Bastiaan Van Loenen. Dutch NGII on course: A practical approach on implementing a vision - the Netherlands. FIG XXII international Congress, Washington DC, USA, Apr 19-26, 2002.

8. Blakemore, M.; McKeever, L. Users of official European statistical data - investigating information needs. Journal of Librarianship and Information Science, No 33 (2), 2001, p. 59-67.

9. Longhorn, R.; Blakemore, M. Re-visiting the valuing and pricing of digital Geographic Information. Journal of Digital Information, Vol 4, Issue 2, 2004 (Web journal).

10. Inspire - Contribution to the extended impact assessment of INSPIRE - 24/9/2003 (draft).

Cindy Pubellier. BSc, CEO of Geolines, France.

Geolines, 5 Place des Brugnauts, 92220 Bagneux, France

$(\mathrm{Ph}+33$ (0) 663719162, Fax: +66 (0) 145366872) e-mail cpubellier@geolines.tk, Web site: www.geolines.tk.

Prior to setting up Geolines, chief technological executive and vice president of IDS, France. More than 15 years of experience in the GIS arena in various countries. A land surveyor, earned her BSc (Honours) degree in surveying and mapping sciences in London. Technical manager of automatic mapping production for Michelin, where she supervised the first digital maps and atlases of Michelin 